\title{
The Probability distribution of a Simple Stochastic Infection and Recovery Processes
}

\author{
Olabisi O. Ugbebor ${ }^{1}$, Joshua O. Okoro ${ }^{2}$ \\ ${ }^{I}$ (Department of Mathematics, University of Ibadan, Ibadan, Nigeria.) \\ ${ }_{2}^{2}$ Industrial Mathematics, Physical Science Department College of Science and Engineering Landmark \\ University, Omu-Aran, Kwara, Nigeria)
}

\begin{abstract}
It is true that when infections occur there might not be recovery. In this case either infections will occur or will do not occur. When measures are put in place to reduce the rate of infection, there might be a tendency for this rate to have an effect on the growth of the infection. When this rate is not checked the whole population might get infected with time. In this work we use the birth-death process to describe a simple but classical infection processes, recovery processes, infection and recovery processes and finally, infection and recovery with immigration processes. For each of these processes explicit formulas are derived for their probability distributions and moment generating functions. We formulate a general infection and recovery process. The conditions for existence of a unique stationary probability for this general infection and recovery process is stated. A density-dependent infection and recovery process is formulated. A quasi-stationary probability distribution is defined, where the process is conditioned on non-extinction. We note here that the infection process has Negative Binomial Distribution and recovery process has a Binomial Distribution.
\end{abstract}

Keywords: Generating Functions, Kolmogorov Differential Equations, Markov Property, Method of

Characteristics

Subject Classification: MSC 2010 60G05, 60G20, 62H10.

\section{Introduction}

When considering a random experiment with sample space, say $\Omega$, we define a random variable $X_{t}(\omega)$ as a single valued function that maps or assigns a real number called the value of $X_{t}(\omega)$ to each sample point $t$ of $\Omega$. A probability space consist of three part $(\Omega, \mathcal{F}, \mathcal{P})$, where $\Omega$ is the sample space, $\mathcal{F}$ is the event space and $\mathcal{P}$ maps events to the interval $[0,1]$. Distribution functions and probability distribution function is a rule that helps you describe the spread of random variable. Example of such maps, assignment or rule is the Probability distribution function or the cumulative distribution function (CDF) of a real-valued variable $X$ at $x$. The probability distribution function may be design for discrete and continuous random variable respectively. A well-known probability mass function for a discrete random variable are for Binomial and Negative Binomial random variable which are given below;

$$
\begin{aligned}
& p_{X}(k)=p_{X}(X=k)=\left(\begin{array}{l}
n \\
k
\end{array}\right) p^{k}(1-p)^{n-k}, \\
& \text { Where } 0 \leq p \leq 1 \text { and } \\
& p_{X}(x)=p(X=x)=\left(\begin{array}{l}
x-1 \\
k-1
\end{array}\right) p^{k}(1-p)^{x-k}, \\
& \text { Where } 0 \leq p \leq 1
\end{aligned}
$$

respectfully.

The mean for a binomial random variable is given by $n p$, while that of negative binomial is given by $\frac{r}{p}$, with variance $n p q$ and $\frac{r(1-p)}{p^{2}}$ respectively, while the moment generating function $M_{X}(t)$ for the binomial and negative binomial distribution is given as $\left(q+p e^{t}\right)^{n}$ and $\left(\frac{p e^{t}}{1-(1-p) e^{t}}\right)^{r}$ respectfully. If $t<\log \left(\frac{1}{(1-p)}\right)$ i.e, $T=\left\{t \in \mathbb{R}: t<\log \left(\frac{1}{(1-p)}\right)\right\}$.

A collection of random variable $\left\{X_{t}(s): t \in T, s \in S\right\}$, where $T$ is some index set and $S$ is the common sample space of the random variable is called a stochastic process. Now, for each fixed $t \in T, X_{t}(s)$ denotes a single random variable defined on $S$, also for each fixed $s \in S, X_{t}(s)$ corresponds to a function defined on $T$ that is called a sample path or a stochastic realization of the process. However for the collection random variable $\left\{X_{t}\left(s_{i}\right): t \in T, s_{i} \in S_{i}\right\}$, where $i=1,2, \ldots, n$ for all $S_{i} \subseteq \Omega$, in this case $T$ is some index set. One of the classification of random processes is Markov Processes. When $P\left\{X\left(t_{n-1}\right) \leq x_{n+1} \mid X\left(t_{1}\right) \leq\right.$ $\left.x_{1}, X\left(t_{2}\right) \leq x_{2}, \ldots, X\left(t_{n}\right) \leq x_{n}\right\}=P\left\{X\left(t_{n-1}\right) \leq x_{n+1} \mid X\left(t_{n}\right) \leq x_{n}\right\}, \quad$ whenever $t_{1}<t_{2}<, \ldots,<t_{n}<t_{n-1}$, such that $(X(t), t \in \boldsymbol{T})$ is a random variable, such a random variable possesses a Markov Process. 
Our interest in this work is to show the probability distribution function of Infection and recovery processes. we formulate a general infection and recovery process. Then the conditions for existence of a unique stationary probability for this general infection and recovery process is stated. It is shown that if the process is nonexplosive, then the general infection and recovering process converges to this stationary probability distribution. Simple but classical infection and recovery processes are presented such as: infection, recovery, infection and recovery, and infection and recovery with immigration processes. Explicit formulas are derived for the moment generating functions. In addition, for the simple infection and simple recovery processes, explicit formulas are derived for their probability distributions. Queueing processes are taken as important application of infection and recovery processes, where infection and recovery are arrivals and departures in the system. A positive stationary probability distribution may not exist for many infection and recovery processes in biology because the zero state (extinction) is absorbing. For such types of processes, the probability of extinction and the expected time until population extinction are investigated. Example of an infection and recovery process with an absorbing state at zero is logistic growth of infection. This density-dependent infection and recovery process is formulated. A quasi-stationary probability distribution is defined, where the process is conditioned on nonextinction. two types of processes that have not been considered previously, an explosive infection process and a nonhomogeneous infection and recovery process.

A good book for stochastic processes with applications to biology is the classic textbook by Bailey, which he title; The Elements of Stochastic Processes with Applications to the Natural Sciences, which has been referenced frequently since its initial publication in 1964.

In his book he said suppose that the random variable $X$ has a m.g.f. $M_{X}(t)$, it can be shown that the m.g.f. of $Y=X-\mu_{X}$ is $M_{Y}(t)=e^{-\mu_{X} t} M_{X}(t)$ and that $M_{Y}^{k}(0)=E\left(Y^{k}\right)=E\left[\left(X-\mu_{X}\right)^{k}\right]$ gives the kth moment of $X$ about its mean [1]. A good book for probability theory is the book by [2]. Another one for probability theory and probability models is the book by [3]. Other references on the basic theory of probability and statistics include [4]. For a more general proof based on characteristic functions, see [5] or [6]. [7], and [8]. The history of the development of the theory of stochastic process began with the study of biological as well as physical problems. According to [9]. One of the first occurrences of a Markov chain may have been in explaining rainfall patterns in Brussels by Quetelet in 1852. The simple branching process was invented by Bienaym'e in 1845 to compute the probability of extinction of a family surname. In 1910, Rutherford and Geiger and the mathematician Bateman described the disintegration of radioactive substances using a Poisson process. In 1905, Einstein described Brownian motion of gold particles in solution, and in 1900, Bachelier used this same process to describe bond prices [9]. The simple birth and death process was introduced by McKendrick in 1914 to describe epidemics. Gibbs in 1902 used nearest-neighbor models to describe the interactions among large systems of molecules [9]. Stochastic processes are now used to model many different types of phenomena from a variety of different areas, including biology, physics, chemistry, finance, economics, and engineering. [10], simulate the simple birth process show that that the random variable for the inter-event time is exponentially distributed. He simulates the time $h \in H$, by mapping it into $U(0,1)$. The function subroutine RAND in the FORTRAN [11] program is a pseudo-random number generator and is based on the recursion relation $y_{n}+$ $1=\left(A y_{n}\right) \bmod M$, where $R A N D=y_{n}+1 / M \in[0,1)$ and the modulus $M=2^{31}-1$ is a Mersenne prime. The term "rand" in the MATLAB program is a built-in MATLAB function for a uniform random number generator on $[0,1]$. A birth and death process with immigration was developed by [12] based on a spatially implicit patch model. in the stochastic formulation by [12], it is assumed that $X(t)$ is the random variable for the number of patches occupied at time $t$. If $X(t)=i$, then the birth and death rates will be denoted as $\lambda_{i}=$ birth rate and $\mu_{i}=$ death rate (see, e.g., [1]; [13]; [14]; [15]; [16]. For a more thorough but elementary introduction to queueing systems, please consult [17], [8], or [16] the distribution can be assumed to be approximately normal or lognormal, referred to as moment closure assumptions (e.g., [18]; [19]).

\section{General infection and recovery Process}

Let $\Delta I(t)$ denote the change in state of the stochastic process from $t$ to $t+\Delta t$. That is, $\Delta I(t)=$ $I(t+\Delta t)-I(t)$. If $X(t)=i$, then the infection and recovery rates will be denoted as $\lambda_{i}=$ infection rate and $\mu_{i}=$ recovery rate. For the general birth and death process see [1], [13], [14], [16]. The continuous-time infection and recovery Markov chain $\{I(t): t \in[0, \infty)\}$ may have either a finite or infinite state space $\{0,1,2, \ldots, N\}$ or $\{0,1, \ldots\}$. Assume the infinitesimal transition probabilities for this process are;

$$
p_{i+j, i}(\Delta t)=\operatorname{Prob}\{\Delta I(t)=j \mid I(t)=i\}= \begin{cases}\lambda_{i} \Delta t+o(\Delta t), & j=1 \\ \mu_{i} \Delta t+o(\Delta t), & j=-1 \\ o(\Delta t), & j=0 \\ 0, & j \neq-1,0,1\end{cases}
$$

for $\Delta t$ sufficiently small, where $\lambda_{i} \geq 0, \mu_{i} \geq 0$ for $i=0,1,2, \ldots$ and $\mu_{0}=0$. It is often the case that $\lambda_{0}=0$, except when there is immigration. The initial conditions are $p_{j i}(0)=\delta_{j i}$, where $P(0)=\left(p_{j i}(0)\right)$ is the identity matrix $P(0)=I$. In a small interval of time $\Delta t$, at most one change in state can occur, either a infection,

DOI: $10.9790 / 5728-1301032029 \quad$ www.iosrjournals.org $21 \mid$ Page


$i \rightarrow i+1$ or a recovering, $i \rightarrow i-1$. From (3), we can derive the forward Kolmogorov differential equations for $p_{j i}(t)$. Now consider the transition probability $p_{j i}(t+\Delta t)$, for $\Delta t$ sufficiently small, we have;

$$
\begin{aligned}
& p_{j i}(t+\Delta t)=p_{j-1, i}(t)\left[\lambda_{j-1} \Delta t+o(\Delta t)\right]+p_{j+1, i}(t)\left[\mu_{j+1} \Delta t+o(\Delta t)\right] \\
& +p_{j i}(t)\left[1-\left(\lambda_{j}+\mu_{j}\right) \Delta t+o(\Delta t)\right]+\sum_{\substack{k \neq-1,0,1 \\
\infty}}^{\infty} p_{j+k, i}(t) o(\Delta t) \\
& =p_{j-1, i}(t) \lambda_{j-1} \Delta t+p_{j+1, i}(t) \mu_{j+1} \Delta t+p_{j i}(t)\left[1-\left(\lambda_{j}+\mu_{j}\right) \Delta t\right]+o(\Delta t)
\end{aligned}
$$

which holds for all $i$ and $j$ in the state space with the exception of $j=0$ and $j=N$ (if the population size is finite). If $j=0$, then $p_{0 i}(t+\Delta t)=p_{1 i}(t) \mu_{1} \Delta t+p_{0 i}(t)\left[1-\lambda_{0} \Delta t\right]+o(\Delta t)$. If $j=N$ is the maximum population size, then

$$
p_{N, i}(t+\Delta t)=p_{N-1, i}(t) \lambda_{N-1} \Delta t+p_{N, i}(t)\left[1-\mu_{N} \Delta t\right]+o(\Delta t) .
$$

If $j=N$ is the maximum population size, then

$$
p_{N, i}(t+\Delta t)=p_{N-1 . i}(t) \lambda_{N-1} \Delta t+p_{N, i}(t)\left[1-\mu_{N} \Delta t\right]+o(\Delta t),
$$

where $\lambda_{N}=0$ and $p_{k, N}(t)=0$ for $k>N$. Subtracting $p_{j i}(t), p_{0 . i}(t)$, and $p_{N, i}(t)$ from equation (4), (5), and (6) respectively, dividing by $\Delta t$ and taking the limit as $\Delta t \rightarrow 0$, yields the forward Kolmogorov differential equations for the general birth and death process,

$$
\begin{gathered}
\frac{d p_{j i}(t)}{d t}=\lambda_{j-1} p_{(j-1), i}(t)-\left(\lambda_{j}+\mu_{j}\right) p_{j i}(t)+\mu_{j+1} p_{j+1, i}(t) \\
\frac{d p_{0 i}(t)}{d t}=-\lambda_{0} p_{0 i}(t)+\mu_{1} p_{1 i}(t)
\end{gathered}
$$

for $i \geq 0$ and $j \geq 1$. For a finite state space, the differential equation for $p_{N, t}(t)$ is $\frac{d p_{N, i}(t)}{d t}=\lambda_{N-1} p_{(N-1), i}(t)-\mu_{N} p_{N i}(t)$.

which can be written in matrix form to give the forward Kolmogorov differential equations which are $\frac{d P}{d t}=Q P$ , where $Q$ is the generator matrix.

\section{Theorem 0.1}

Suppose the CTMC $\{I(t): t \in[0, \infty)\}$ is a general continuous-time infection and recovery Markov chain has infection and recovery rates, $\lambda_{i-1}=\lambda$ and $\mu_{i}=i \mu$, If the state space is infinite then a unique positive stationary probability distribution $\pi$ exists iff $\mu_{i}>0$ and $\lambda_{i-1}>0$ which is given by

for $i=1,2, \ldots$

$$
\pi_{i}=\frac{\left(\frac{\lambda}{\mu}\right)^{i}}{i !} e^{-\frac{\lambda}{\mu}}
$$

\section{Theorem 0.2}

Suppose a continuous-time infection and recovery Markov chain has infection and recovery rates $\mu_{i}=\mu>0$, $i=1,2, \ldots$, and $\lambda i=\lambda>0, i=0,1,2, \ldots$, where $p+q=1$. The embedded Markov chain is a semi-infinite random walk model with reflecting boundary conditions at zero. The chain has a unique stationary probability distribution iff

$$
\sum_{j=1}^{\infty}\left(\frac{\lambda}{\mu}\right)^{j}<\infty
$$

Iff $\lambda<\mu$.

The stationary probability distribution is a geometric probability distribution, $\pi_{0}=\frac{\mu-\lambda}{\mu}$ and $\pi_{i}=\left(\frac{\lambda}{\mu}\right)^{i} \pi_{0}$ for $i=0,1,2, \ldots$.

\section{Theorem 0.3}

Let $\{I(t): t \in[0, \infty)\}$ be a nonexplosive, positive recurrent, and irreducible CTMC with transition matrix $P(t)=\left(p_{i j}(t)\right)$ and generator matrix $Q=\left(q_{i j}\right)$. Then there exists a unique positive stationary probability distribution $\pi, Q \pi=0$, such that $\lim _{n \rightarrow \infty} p_{i j}(t)=\pi_{i}, i, j=1,2, \ldots$ It follows that the mean recurrence time can be computed from the stationary distribution:

$$
\pi_{i}=-\frac{1}{q_{i i} \mu_{i i}}>0 .
$$




\section{The Simple Infection}

In the simple infection process, the only event is infection. Let $I(t)$ represent the population size at time $t$ and $I(0)=I$, so that $p_{i}(0)=\delta i I$. Since the only event here is infection, the population size of infection can only increase in size. For $\Delta t$ sufficiently small, the transition probabilities are

$$
p_{i+j, i}(\Delta t)=\operatorname{Prob}\{\Delta I(t)=j \mid I(t)=i\}= \begin{cases}\lambda i \Delta t+o(\Delta t), & j=1 \\ 1-\lambda i \Delta t+o(\Delta t), & j=0 \\ o(\Delta t), & j \geq 2 \\ 0, & j<0\end{cases}
$$

The probabilities pi(t) $=\operatorname{Prob}\{I(t)=i\}$ are solutions of the forward Kolmogorov differential equations, $\frac{d p}{d t}=Q p, \quad$ where $\quad \frac{d p_{i}(t)}{d t}=\lambda(i-1) p_{i-1}(t)-\lambda i p_{i}(t), i=N, N+1, \ldots, \quad$ and $\quad \frac{d p i(t)}{d t}=0$, for $\quad i=$ $0,1, \ldots, N-1$. Then $\frac{d p_{N}}{d t}=-\lambda N p_{N}$ so that the solution is $p N(t)=e^{-\lambda N t}$, for $i<N$

\subsection{Generating Functions for the Simple Infection Process}

To derive the partial differential equation for the p.g.f., multiply the differential equations by $z^{i}$ and sum over $i$, we have; $\frac{\partial P(z, t)}{\partial t}=\lambda z(z-1) \frac{\partial P}{\partial t}$. The initial condition is $P(z, 0)=z^{N}$. The partial differential equation for the m.g.f. can be derived by a change of variable. Let $z=e^{\theta}$. Then $P\left(e^{\theta}, t\right)=M(\theta, t)$ and $\frac{\partial P}{\partial z}=\frac{1}{z} \frac{\partial M}{\partial \theta}$. so that the m.g. $f$ is a solution of the following partial differential equation: $\frac{\partial M(z, t)}{\partial t}=\lambda\left(e^{\theta}-1\right) \frac{\partial M}{\partial \theta}$ with initial condition $M(\theta, 0)=e^{N \theta}$.Rewriting the differential equation for $M(\theta, t)$, we have;

$\frac{\partial M}{\partial t}+\lambda\left(1-e^{\theta}\right) \frac{\partial M}{\partial \theta}=0$. The method of characteristics is applied to find the solution $M(\theta, t)$. Hence we have;

$\frac{\partial M}{\partial \tau}=\frac{\partial M}{\partial t} \frac{d t}{d \tau}+\frac{\partial M}{\partial \theta} \frac{\partial \theta}{\partial \tau}$. Along characteristic curves, $s$ and $\tau, t(s, \tau), \theta(s, \tau)$, and $M(s, \tau)$ are solutions of $\frac{d t}{d \tau}=1, \frac{\partial \theta}{\partial \tau}=\lambda\left(1-e^{\theta}\right), \frac{\partial M}{\partial \tau}=0$, with initial conditions $t(s, 0)=0, \theta(s, 0)=s$, and $M(s, 0)=e^{N s}$.

Separating variables and simplifying leads to $\frac{e^{-\theta} \partial \theta}{e^{-\theta}-1}=\lambda d \tau$. Integrating yields the relations, $=\tau, \ln \left(e^{-\theta}-\right.$ $1)=-\lambda \tau+c$, and $M(s, \tau)=e^{N s}$. Applying the initial condition $\theta(s, 0)=s$, the second expression can be written as $\ln \left(e^{-\theta}-1\right)-\ln \left(e^{-s}-1\right)=-\lambda \tau$ therefore we have $\left(e^{-\theta}-1\right)=\left(e^{-s}-1\right) e^{-\lambda \tau}$.

Finally, the solution $M$ must be expressed in terms of the original variables $\theta$ and $t$. Using the preceding formulas, $e^{-s}$ can be expressed in terms of $\theta$ and $t, e^{-s}=1-e^{\lambda t}\left(1-e^{-\theta}\right)$. Since $e^{N s}=\left[e^{-s}\right]^{-N}$, the $m . g . f$. for the simple infection process is

$$
M(\theta, t)=\left[1-e^{\lambda t}\left(1-e^{-\theta}\right)\right]^{-N}
$$

The p.g.f can be found directly from the m.g.f. by making the change of variable, $\theta=\ln z$,

Letting $p=e^{-\lambda t}$ and $q=1-e^{-\lambda t}$, the $p . g . f$. is

$$
P(z, t)=\left(\frac{z e^{-\lambda t}}{\left[1-z\left(1-e^{-\lambda t}\right)\right]}\right)^{\mathrm{N}}
$$

$$
P(z, t)=\frac{(p z)^{N}}{(1-z q)^{N}}
$$

The p.g.f. and m.g.f. for the simple infection process correspond to a negative binomial distribution. The probabilities $p_{i}(t)$ in (10) can be written as

$$
p_{i+N}(t)=\left(\begin{array}{c}
N+i-1 \\
i
\end{array}\right) p^{N} q^{i}, \quad i=0,1,2, \ldots
$$

Let $i+N=n$ and replace $p$ and $q$ by $e^{-\lambda t}$ and $1-e^{-\lambda t}$, respectively,

$$
\begin{aligned}
& i=n-N \\
& p_{n}(t)=\left(\begin{array}{c}
n-1 \\
n-N
\end{array}\right) e^{-\lambda N t}\left(1-e^{-\lambda t}\right)^{n-N}, \\
& n=N, N+1, N+2, \ldots
\end{aligned}
$$

Now for $N=a$, the initial infectious population, we have;
\[ p_{n}(t)=\left(\begin{array}{c}n-1 \\ n-a\end{array}\right) e^{-b a t}\left(1-e^{-b t}\right)^{n-a} \]
$=\left(\begin{array}{l}n-1 \\ a-1\end{array}\right) e^{-b N t}\left(1-e^{-b t}\right)^{n-a}, \quad$ for $\quad n=a, a+1, a+2, \ldots$ 


\section{The Simple Recovery Process}

In the simple recovery process, the only event is a recovery. Let $X(0)=N$. The infinitesimal transition probabilities are

$$
\begin{gathered}
p_{i+j, i}(\Delta t)=\operatorname{Prob}\{\Delta I(t)= \\
j \mid I(t)=i\}= \begin{cases}\mu i \Delta t+o(\Delta t), & j=-1 \\
1-\mu i \Delta t+o(\Delta t), & j=0 \\
o(\Delta t), & j \leq-2 \\
0, & j>0\end{cases}
\end{gathered}
$$

Since the process begins in state $N$, the state space is $\{0,1,2, \ldots, N\}$. The probabilities $p_{i}(t)=\operatorname{Prob}\{X(t)=$ $i\}$ are solutions of the forward Kolmogorov differential equations, $\frac{d p}{d t}=Q p$, where by the forward Kolmogorov equations we have

$$
\begin{gathered}
\frac{d p_{i}(t)}{d t}=\mu(i+1) p_{i+1}(t)-\mu i p_{i}(t), \\
\frac{d p_{N}(t)}{d t}=-\mu N p_{N}(t),
\end{gathered}
$$

$i=0,1, \ldots, N-1$, with initial conditions $p_{i}(0)=\delta_{i N}$. It can be easily seen that zero is an absorbing state and the unique stationary probability distribution is $\pi=(1,0,0, \ldots, 0)$.

The generating function technique and the method of characteristics are used to find the probability distribution. Multiplying the forward Kolmogorov equations by $z^{i}$ and summing over $i$, the partial differential equation for the p.g.f. is

$$
\begin{gathered}
\frac{\partial P(z, t)}{\partial t}=\mu \sum_{i=0}^{N}(i+1) p_{i+1} z^{i}-\mu \sum_{i=1}^{N} i p_{i} z^{i} \\
=\mu(1-z) \frac{\partial P}{\partial z}
\end{gathered}
$$

The initial condition is $P(z, 0)=z^{N}$.

The partial differential equation for the m.g.f. can be derived by a change of variable. Let $z=e^{\theta}$, hence, $\ln z=\theta, \frac{d \theta}{d z}=\frac{1}{z}=\frac{1}{e^{\theta}}=e^{-\theta}$, then $P\left(e^{\theta}, t\right)=M(\theta, t)$ and

$$
\frac{\partial P}{\partial z}=\frac{\partial M}{\partial \theta} \frac{d \theta}{d z}=\frac{1}{z} \frac{\partial M}{\partial \theta},
$$

so that the m.g.f is a solution of the following partial differential equation:

with initial condition $M(\theta, 0)=e^{N \theta}$. Hence we can have;

$$
\frac{\partial M(z, t)}{\partial t}=\mu\left(1-e^{\theta}\right) \frac{\partial P}{\partial z}=\mu\left(e^{-\theta}-1\right) \frac{\partial M}{\partial \theta}
$$

$$
\frac{\partial M}{\partial \tau}=\frac{\partial M}{\partial t} \frac{d t}{d \tau}+\frac{\partial M}{\partial \theta} \frac{\partial \theta}{\partial \tau}=0
$$

Along characteristic curves, $s$ and $\tau, t(s, \tau), \theta(s, \tau)$, and $M(s, \tau)$ are solutions of $\frac{d t}{d \tau}=1, \frac{\partial \theta}{\partial \tau}=\mu\left(1-e^{-\theta}\right)$, $\frac{\partial M}{\partial \tau}=0$. Rewriting the differential equation for $(\theta, t), \frac{\partial M}{\partial t}+\mu\left(1-e^{-\theta}\right) \frac{\partial M}{\partial \theta}=0$. The method of characteristics is applied to find the solution $M(\theta, t)$ with initial conditions $t(s, 0)=0, \theta(s, 0)=s$, and $M(s, 0)=e^{N s}$. Separating variables and simplifying leads to $\frac{\partial \theta}{\left(1-e^{-\theta}\right)}=\mu d \tau$ or $\frac{e^{\theta} \partial \theta}{e^{\theta}-1}=\mu d \tau$

Integrating yields the relations at $=\tau, \ln \left(e^{\theta}-1\right)=\mu \tau+c$, and $M(s, \tau)=e^{N s}$.

Applying the initial condition $\theta(s, 0)=s$, the second expression can be written as $\ln \left(e^{\theta}-1\right)-\ln \left(e^{s}-1\right)=$ $\mu \tau$

$\left(e^{\theta}-1\right)=\left(e^{s}-1\right) e^{\mu \tau}$

$$
\begin{gathered}
\ln \left(\frac{\left(e^{\theta}-1\right)}{\left(e^{S}-1\right)}\right)=\mu \tau \\
\left(\frac{\left(e^{\theta}-1\right)}{\left(e^{S}-1\right)}\right)=e^{\mu \tau}
\end{gathered}
$$

$$
\begin{gathered}
e^{s}-1=e^{-\mu \tau}\left(e^{\theta}-1\right) \\
e^{s}=1+e^{-\mu \tau}\left(e^{\theta}-1\right)=1-e^{-\mu \tau}\left(1-e^{\theta}\right)
\end{gathered}
$$


Finally, the solution $M$ must be expressed in terms of the original variables $\theta$ and $t$. Using the preceding formulas, $e^{-s}$ can be expressed in terms of $\theta$ and $t, e^{s}=1-e^{-\mu t}\left(1-e^{\theta}\right)$. Since $e^{N s}=\left[1-e^{-\mu t}(1-\right.$ $\left.\left.e^{\theta}\right)\right]^{N}$, the $m . g . f$. for the simple recovery process is

$$
M(\theta, t)=\left[1-e^{-\mu t}\left(1-e^{\theta}\right)\right]^{N}
$$

The p.g.f can be found directly from the m.g.f. by making the change of variable, $\theta=\ln z$,

$$
P(z, t)=\left[1-e^{-\mu t}(1-z)\right]^{N}=\left[1-e^{-\mu t}+e^{-\mu t} z\right]^{N}
$$

Let $p=e^{-\mu t}$ and $q=1-e^{-\mu t}$. Then the p.g.f. has the form $(z, t)=(q+p z)^{N}$, corresponding to a binomial distribution, $b(N, p)$. The probabilities;

for $i=0,1, \ldots, N$.

$$
p_{i}(t)=\left(\begin{array}{c}
N \\
i
\end{array}\right) p^{i} q^{N-i}=\left(\begin{array}{c}
N \\
i
\end{array}\right) e^{-i \mu t}\left(1-e^{-\mu t}\right)^{N-i}
$$

\section{The Simple Infection And Recovering}

In the simple birth and death process, an event can be a birth or a death. Let $I(0)=I_{0}$. The infinitesimal transition probabilities are

$$
\begin{gathered}
p_{i+j, i}(\Delta t)=\operatorname{Prob}\{\Delta I(t)= \\
j \mid I(t)=i\}=\left\{\begin{array}{cl}
\lambda i \Delta t+o(\Delta t), & j=-1 \\
\lambda i \Delta t+o(\Delta t), & j=1 \\
1-(\lambda+\mu) i \Delta t+o(\Delta t), & j=0 \\
o(\Delta t), & j \neq-1,0,1 .
\end{array}\right.
\end{gathered}
$$

The forward Kolmogorov differential equations are

$$
\begin{gathered}
\frac{d p_{i}(t)}{d t}=\lambda(i-1) p_{i-1}(t)+\mu(i+1) p_{i+1}(t)-(\lambda+\mu) i p_{i}(t) \\
d p_{0}(t) d t=\mu p_{1}(t)
\end{gathered}
$$

for $i=1,2, \ldots$ with initial conditions $p_{i}(0)=\delta_{i N}$. As in the simple death process, $\lambda_{0}=0$, so that zero is an absorbing state and $\pi=(1,0,0, \ldots)^{t r}$ is the unique stationary probability distribution. Applying the generating function technique and the method of characteristics from the Appendix for Chapter 6 of [20], the moment generating (m.g.f.) function and probability generating function (p.g.f.) is ;

$\left.M(\theta, t)=\left(\frac{e^{t(\mu-\lambda)}\left(\lambda e^{\theta}-\mu\right)-\mu\left(e^{\theta}-1\right.}{e^{t(\mu-\lambda)}\left(\lambda e^{\theta}-\mu\right)-\lambda\left(e^{\theta}-1\right)}\right)\right)^{I_{0}}$ and $P(z, t)=\left(\frac{e^{t(\mu-\lambda)}(\lambda z-\mu)-\mu(z-1)}{e^{t(\mu-\lambda)}(\lambda z-\mu)-\lambda(z-1)}\right)^{I_{0}}$ respectfully if the rate of infection is not equal to the rate of recovery.

$M(\theta, t)=\left(\frac{1-(\lambda t-1)\left(e^{\theta}-1\right)}{1-\lambda t\left(e^{\theta}-1\right)}\right)^{I_{0}}$ and $P(z, t)=\left(\frac{1-(\lambda t-1)(z-1)}{1-\lambda t(z-1)}\right)^{I_{0}}$ if the rate of infection is equal to the rate of recovery

The $p_{i}(t)$ is not easy as it was for the simple infection and recovery processes, this is made more, since functions cannot be associated with a well-known probability distribution, but we know that;

$P(z, t)=\sum_{i=0}^{\infty} p_{i}(t) z^{i}$ and $p_{i}(t)=\left.\frac{1}{i} \frac{\partial^{i} P}{\partial z^{i}}\right|_{z=0}$. Using computer software, we can find the terms of the series expansion. $p_{0}(t)=P(0, t)$ is the first term of the series expansion. For $\lambda \neq \mu$,

$p_{0}(t)=P(0, t)=\left(\frac{\mu-\mu e^{(\mu-\lambda) t}}{\lambda-\mu e^{(\mu-\lambda) t}}\right)^{I_{0}}$ and for $\lambda=\mu ; p_{0}(t)=\left(\frac{\lambda t}{1-\lambda t}\right)^{I_{0}}$. As $t \rightarrow \infty$, the probability of extinction $p_{0}(t)$ will be;

$$
p_{0}(t)=p_{0}(\infty)=\lim _{t \rightarrow \infty} p_{0}(t)= \begin{cases}1, & \text { if } \lambda \leq \mu \\ \left(\frac{\mu}{\lambda}\right)^{I_{0}}, & \text { if } \lambda>\mu\end{cases}
$$

The mean and variance of the simple infection and recovery process can be derived from the generating functions.

And

$$
m(t)= \begin{cases}I_{0} e^{(\lambda-\mu) t}, & \text { if } \lambda \neq \mu \\ \mathrm{I}_{0}, & \text { if } \lambda=\mu\end{cases}
$$

$$
\sigma^{2}(t)= \begin{cases}I_{0} \frac{(\lambda+\mu)}{(\lambda-\mu)} e^{(\lambda-\mu) t}\left(e^{(\lambda-\mu) t}-1\right), & \text { if } \lambda \neq \mu \\ 2 \mathrm{I}_{0} \lambda t, & \text { if } \lambda=\mu\end{cases}
$$

From equation (14) and (15), we see that correspond to exponential growth, when $\lambda>\mu$ and exponential decay when $\lambda<\mu$. 


\section{The Simple Birth and Death with Immigration}

When there an immigration in the compartment of infected, the rate of immigration will added to the rate of general infection; whatever it is. Suppose the immigration rate is assume constant rate $g$. We let the initial population of infected to be $I_{0}$. Transition probabilities for this process is given below;

$$
\begin{gathered}
p_{i+j, i}(\Delta t)=\operatorname{Prob}\{\Delta I(t)= \\
j \mid I(t)=i\}= \begin{cases}\mu i \Delta t+o(\Delta t), & j=-1 \\
(g+\lambda i) \Delta t+o(\Delta t), & j=1 \\
1-[g+(\lambda+\mu) i] \Delta t+o(\Delta t), & j=0 \\
o(\Delta t), & j \neq-1,0,1 .\end{cases}
\end{gathered}
$$

The forward Kolmogorov differential equations are

$$
\begin{gathered}
\frac{d p_{i}(t)}{d t}=[\lambda(i-1)-g] p_{i-1}(t)+\mu(i+1) p_{i+1}(t)-[(\lambda+\mu) i+g] p_{i}(t) \\
d p_{0}(t) d t=-g p_{0}+\mu p_{1}(t)
\end{gathered}
$$

for $i=1,2, \ldots$ with initial conditions $p_{i}(0)=\delta_{i I_{0}}$. the m.g.f. $M(\theta, t)$ is a solution of

with $M(\theta, 0)=e^{I_{0} \theta}$ as initial condition. [1] gave the solution as

$$
\frac{\partial M}{\partial t}=\lambda\left(e^{\theta}-1\right)+\mu\left(e^{-\theta}-1\right) \frac{\partial M}{\partial \theta}+g\left(e^{\theta}-1\right) M
$$

$$
M(\theta, t)=\frac{(\lambda-\mu)^{\frac{g}{\lambda}}\left[\mu\left(e^{(\lambda-\mu) t}-1\right)-e^{\theta}\left(\mu e^{(\lambda-\mu) t}-\lambda\right)\right]^{I_{0}}}{\left[\left(e^{(\lambda-\mu) t}-\mu\right)-\lambda\left(e^{(\lambda-\mu) t}-1\right) e^{\theta}\right]^{I_{0}+\frac{g}{\lambda}}}
$$

The moments of the probability distribution $I(t)$ can be found by differentiating with respect to $\theta$ and evaluating at $\theta=0$. The mean is

$$
m(t)= \begin{cases}\frac{e^{(\lambda-\mu) t}\left(I_{o} \mu-I_{0} \lambda-g\right)+g}{\lambda-\mu}, & \text { if } \lambda \neq \mu \\ g t+I_{0}, & \text { if } \lambda=\mu\end{cases}
$$

When $\lambda>\mu$, the mean increases exponentially in time and linearly when $\lambda=\mu$. However, in the case $\lambda<\mu$, the mean approaches a constant:

$$
m(\infty)=\frac{g}{\mu-\lambda}
$$

Thus, for the case $\lambda<\mu$, the process is nonexplosive and irreducible.

From

Theorem 0.1 and

Theorem 0.2, it can be shown that the process has a unique positive stationary distribution. [14] shows that the conditions of being nonexplosive, irreducible, and having a positive stationary distribution imply the process is positive recurrent. Then,

Theorem 0.3 implies the limiting distribution exists and equals the stationary distribution.

\section{Result and Conclusion}

The mean and variance for the simple infection process are $m(t)=N e^{\lambda t}$ and $\sigma^{2}(t)=N e^{2 \lambda t}(1-$ $\left.e^{-\lambda t}\right)$. The moments can be calculated directly from one of the generating functions. It is interesting to note that the mean of the simple infection process corresponds to exponential growth with $I_{0}=N$. The variance also increases exponentially with time. 


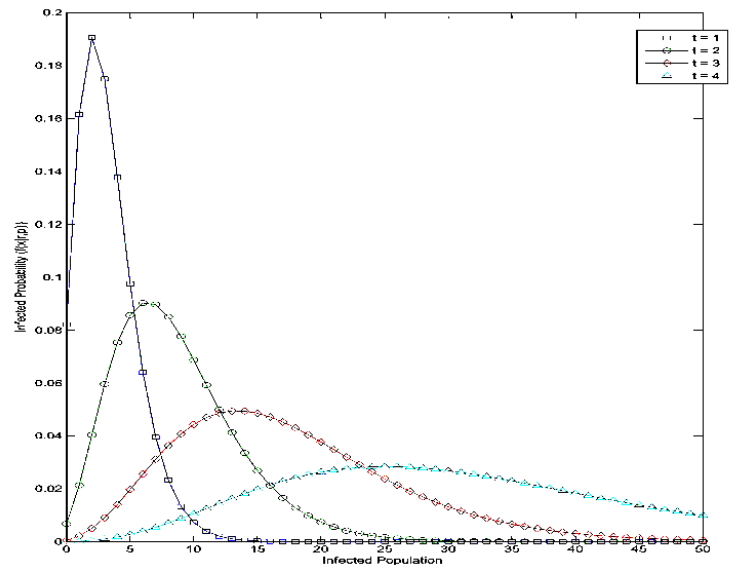

Fig 0.1: Probability distributions of $I(t)$ for the simple infection process for $t=1,2,3,4$

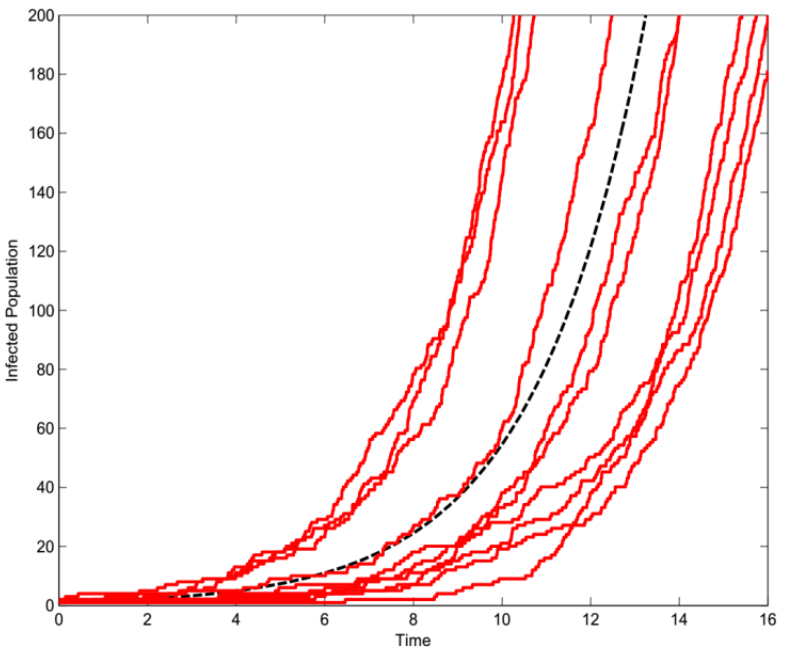

Fig 0.2: Ten sample paths when $\lambda=0.4$ and $I(0)=2$. The mean and variance are $m(t)=2 e^{0.5 t}, \sigma^{2}(t)=$ $2\left(e^{t}-e^{0.5 t}\right)$

The mean and variance of a binomial distribution $b(N, p)$ are $m=N p$ and $\sigma^{2}=N p q \cdot m(t)=N e^{-\mu t}$ and $\sigma^{2}(t)=N e^{-\mu t}\left(1-e^{-\mu t}\right)$.

The mean corresponds to exponential decay. Also, the variance decreases exponentially with time.

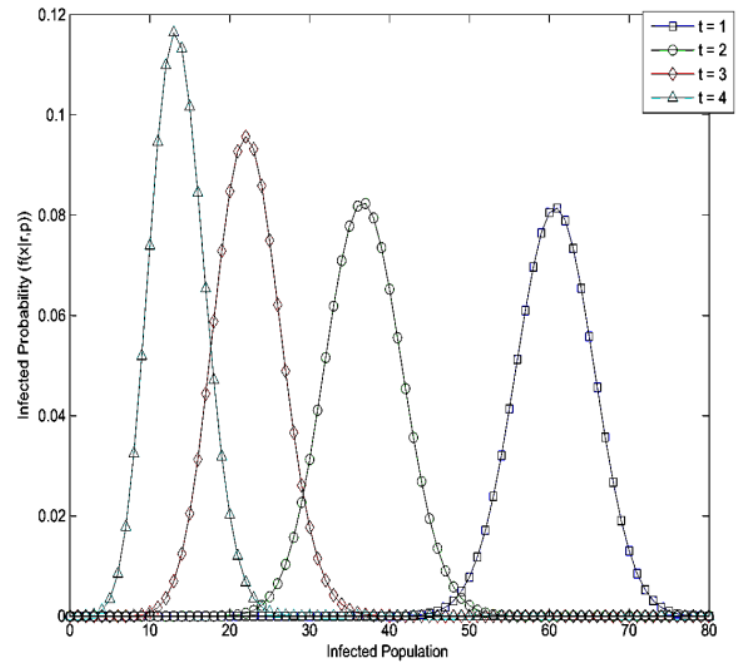


Fig 0.3: Probability distributions of $I(t)$ for the simple death process for $t=1,2,3,4$

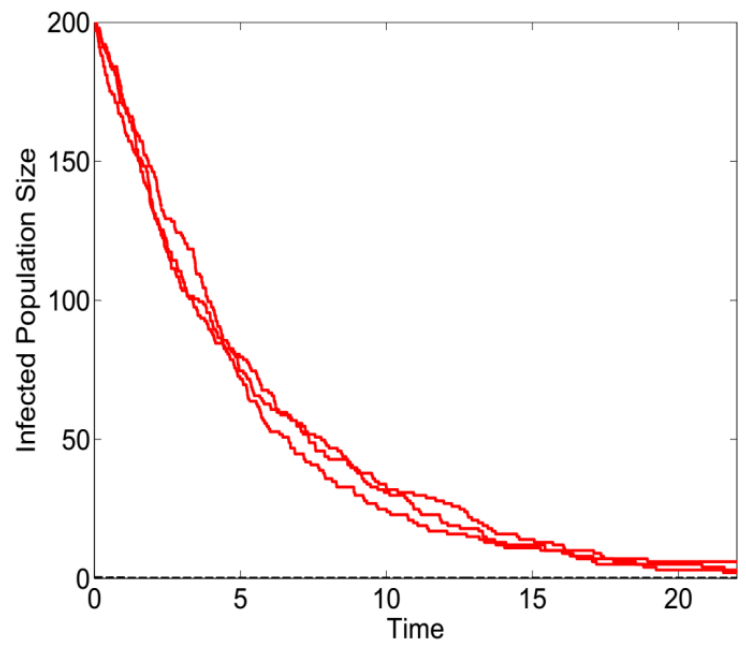

Fig 0.4: three sample paths when $\mu=0.2$ and $I(0)=200$. The mean and variance are $m(t)=200 e^{-0.5 t}$ and $\sigma^{2}(t)=200\left(e^{-0.2 t}-e^{-t}\right)$.

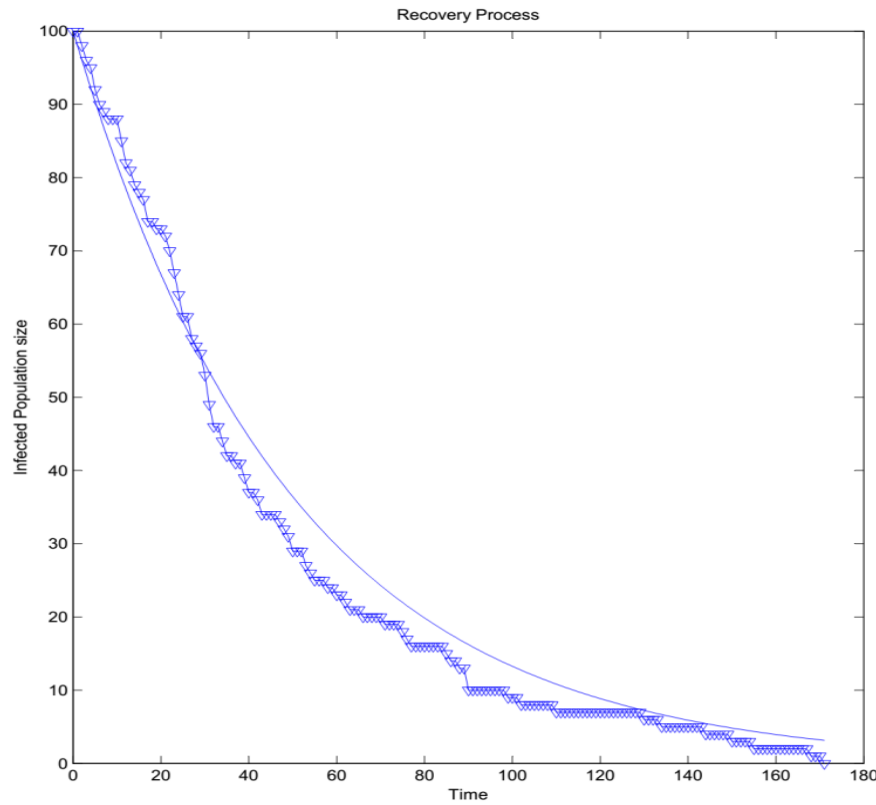

We simulates the 'Recovery process. Starting with a given initial individuals, each having probability $p$ of recovering in each time step, we continue until all have recover. The infected population size is plotted over time and compared to the deterministic model for expected infected population size.

For $\lambda \neq \mu$,

$p_{0}(t)=P(0, t)=\left(\frac{\mu-\mu e^{(\mu-\lambda) t}}{\lambda-\mu e^{(\mu-\lambda) t}}\right)^{I_{0}}$ and for $\lambda=\mu ; p_{0}(t)=\left(\frac{\lambda t}{1-\lambda t}\right)^{I_{0}}$. As $t \rightarrow \infty$, the probability of extinction $p_{0}(t)$ will be;

$$
p_{0}(t)=p_{0}(\infty)=\lim _{t \rightarrow \infty} p_{0}(t)= \begin{cases}1, & \text { if } \lambda \leq \mu \\ \left(\frac{\mu}{\lambda}\right)^{I_{0}}, & \text { if } \lambda>\mu\end{cases}
$$

The mean and variance of the simple infection and recovery process can be derived from the generating functions.

And

$$
m(t)= \begin{cases}I_{0} e^{(\lambda-\mu) t}, & \text { if } \lambda \neq \mu \\ \mathrm{I}_{0}, & \text { if } \lambda=\mu\end{cases}
$$

$$
\sigma^{2}(t)= \begin{cases}I_{0} \frac{(\lambda+\mu)}{(\lambda-\mu)} e^{(\lambda-\mu) t}\left(e^{(\lambda-\mu) t}-1\right), & \text { if } \lambda \neq \mu \\ 2 \mathrm{I}_{0} \lambda t, & \text { if } \lambda=\mu\end{cases}
$$


From equation (14) and (15), we see that correspond to exponential growth, when $\lambda>\mu$ and exponential decay when $\lambda<\mu$.

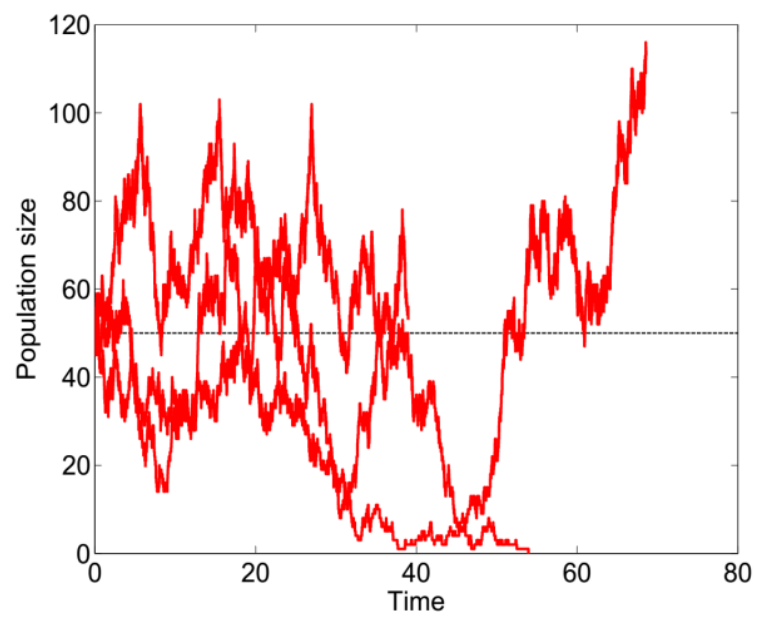

Fig 0.5: Three sample paths for the simple infection and recovery process when $\lambda=1=\mu$ and $I(0)=50$.

\section{References}

[1]. N. T. J. Bailey, The Elements of Stochastic Processes with Applications to the Natural Sciences., New York: John Wiley \& Sons, 1990.

[2]. W. Feller, An Introduction to Probability Theory and Its Applications., vol. 1, 3, Ed., New York.: John Wiley \& Sons, 1968.

[3]. S. M. Ross, A First Course in Probability, Upper Saddle River, N. J.: Pearson Prentice Hall, 2006.

[4]. R. V. Hogg and A. T. Craig, Introduction to Mathematical Statistics, 5, Ed., Upper Saddle River, N. J.: Prentice Hall, 1995.

[5]. H. Cram'er, Mathematical Methods of Statistics, Princeton, N. J.: Princeton Univ. Press, 1945.

[6]. M. J. Schervish, Theory of Statistics, New York, Berlin, Heidelberg.: Springer-Verlag, 1995.

[7]. R. V. Hogg and E. A. Tanis., Probability and Statistical Inference, 6, Ed., Upper Saddle River, N. J.: Prentice Hall, 2001.

[8]. H. P. Hsu, Schaum's Outline of Theory and Problems of Probability, Random Variables, and Random Processes., New York.: McGraw-Hill, 2014

[9]. P. Guttorp, Stochastic Modeling of Scientific Data, London: Chapman \& Hall, 1995.

[10]. E. Renshaw, Modelling Biological Populations in Space and Time Cambridge Studies in Mathematical Biology., Cambridge, U. K.: Cambridge Univ. Press, 1993.

[11]. S. L. Anderson, "Random number generators on vector supercomputers and other advanced architectures," SIAM Review., vol. 32, p. 221-251, 1990.

[12]. D. Alonso and A. McKane, "Extinction dynamics in mainlandisland metapopulations: an N-patch stochastic model.," Bull. Math. Biol., vol. 64, p. 913-958, 2002.

[13]. S. Karlin and H. Taylor, A First Course in Stochastic Processes, New York: Academic Press, 1975.

[14]. J. R. Norris, "Markov Chains. Cambridge Series in Statistical and Probabilistic Mathematics," Cambridge Univ. Press, , pp. Cambridge, U. K., 1997.

[15]. R. B. Schinazi, Classical and Spatial Stochastic Processes., Boston.: Birkh“auser, 1999.

[16]. H. M. Taylor and S. Karlin, An Introduction to Stochastic Modeling, New York.: Academic Press, 1998.

[17]. A. T. Bharucha-Reid, Elements of the Theory of Markov Processes and their Applications., New York: Dover Pub., 1997.

[18]. M. S. Chan and V. S. Isham, "A stochastic model of schistosomiasis immuno-epidemiology," Math. Biosci., vol. 151, p. 179-198, 1998.

[19]. A. L. Lloyd, "Estimating variability in models for recurrent epidemics: assessing the use of moment closure techniques.," Theor. Pop. Biol., vol. 65, pp. 49 - 65, 2004.

[20]. L. J. S. Allen, An Introduction to Stochastic Processes with Applications to Biology, 2, Ed., LUbbock, Texas, USA: CRC Press Taylor \& Francis Group, 2010 